\title{
Per una rilettura kantiana del principio di causalità
}

ANGElo CiCATEllo

Dipartimento di Scienze Umanistiche

Università degli Studi di Palermo

90128 Palermo (Italia)

angelo.cicatello@unipa.it
Abstract: There is no contradiction between Kant's statement that the proposition, "every alteration has its cause," is of no interest to the Critique of Pure Reason because of its dependence on empirical contents ( $\mathrm{KrV}, \mathrm{B}_{3}$ ) and his use of the same proposition as an example of pure a priori knowledge (KrV, B 5). There is only the arduousness and sometimes also the ambiguity of a passage in which Kant attempts to establish a new basis for the validity of the principle of causality.

Keywords: Kant, principles, causality, time, alteration.
Resumen: No hay contradicción entre la afirmación kantiana de que la proposición "toda alteración tiene su causa" no tiene ningún interés para la Crítica de la Razón Pura debido a sus compromisos con los contenidos empíricos (KrV, B 3) y el hecho de que la misma proposición se cita explícitamente como ejemplo de conocimiento puro a priori (KrV, B 5). Solamente hay la dificultad y a veces también la ambigüedad de un paso que Kant, entre otras dificultades, trata de dar en un intento de establecer la validez del principio de causalidad sobre nuevas bases.

Palabras clave: Kant, principios, causalidad, tiempo, alteración. 
U

n equivoco. Così Kant si difende dai rilievi mossigli in una recensione anonima pubblicata nella "Leipziger gelehrte Zeitung" (1787, n. 94) in merito alla palese contraddizione in cui sarebbe incappato nell'Introduzione alla Critica della ragion pura. I termini della questione sono noti e riguardano la distinzione, all'interno delle conoscenze a priori, tra le conoscenze pure, nel senso di non mescolate ad elementi empirici, e quelle il cui contenuto reca, invece, in sé anche qualcosa che è ricavato dall'esperienza. Come esempio di queste ultime, dunque delle presunte conoscenze a priori non pure, Kant cita la proposizione "ogni mutamento ha una causa", con la motivazione che "il mutamento è un concetto che può essere tratto soltanto dall'esperienza"1. Il lettore, tuttavia, non deve fare molta strada, due pagine soltanto, per ritrovare la stessa proposizione citata, però, questa volta come esempio di giudizi puri a priori. Anche qui Kant fornisce una motivazione precisa:

In quest'ultimo principio è così evidente che il concetto stesso di causa contiene in sé il concetto della necessità di una connessione con l'effetto e quello della rigorosa universalità della regola, che esso andrebbe completamente perso se lo si volesse derivare - come ha fatto Hume- dalla frequente associazione di ciò che accade con ciò che lo precede, e dalla conseguente abitudine (dunque, da una necessità semplicemente soggettiva) a collegare delle rappresentazioni ${ }^{2}$.

1. KrV, B 3. Per le opere di Kant si fa riferimento al testo della Akademie-Ausgabe (Kants Gesammelte Schriften, hrsg. von der Preußischen Akademie der Wissenschaften, Berlin 1902 sgg.), indicato con la sigla AA, cui segue immediatamente l'indicazione del numero del volume e del numero di pagina. Fa eccezione la Kritik der reinen Vernunft $(=\mathrm{KrV})$, che viene invece citata nelle pagine della prima e seconda edizione originale (A e B), riportate peraltro nella traduzione italiana qui utilizzata. I. Kant, Critica della ragion pura, tr. it. di C. EsPosito (Bompiani, Milano, 2004).

2. KrV, B 5. L'obiezione mossa a Kant dal recensore anonimo viene anche riportata da H. Waininger, Commentar zur Kants Kritik der reinenn Vernunft, (Spemann, Stuttgart, 1881) 211. Su questa presunta contraddizione è tornato più di recente A. Ferrarin per evidenziare il carattere fuorviante e ingannevole di certe procedu- 
Sul fatto che l'esempio venga usato in modo equivoco non può esservi alcun dubbio, tanto più che lo stesso Kant, in risposta ai rilievi del solerte recensore, ammette che avrebbe potuto prevenire il malinteso, ricorrendo, ad esempio, per le conoscenze pure a priori alla proposizione "ogni contingente ha una causa", così da evitare il riferimento empirico al fenomeno del mutamento ${ }^{3}$. Che tale riferimento possa dar luogo a una commistione indebita dell'ordine delle conoscenze di stretta pertinenza della Critica della ragion pura con il piano delle conoscenze empiriche è, peraltro, attestato in più occasioni nel testo kantiano. Così, si legge ad esempio che

L'Estetica trascendentale non può annoverare tra i suoi dati a priori il concetto di mutamento, poiché non è il tempo stesso che muta, bensì qualcosa che è nel tempo. Per questo si richiede allora la percezione di un qualcosa di esistente e della successione delle sue determinazioni, e dunque si richiede l'esperienza"4.

E ancora, nell'Analitica trascendentale, Kant fa riferimento esplicito al fatto che la causalità di un mutamento in generale presuppone principi empirici e pertanto rimane fuori dai confini di una filosofia trascendentale 5

re dicotomiche kantiane. A. FERRARIN, Come è possibile comprendere i giudizi sintetici a priori, in L. AMOROSO, C. LA RoCCA. A. FERRARIN (a cura di), Critica della ragione e fenomenologia dell'esperienza (ETS, Pisa, 2011) 395-414, 395. In realtà, come cercherò di mostrare, la distinzione tra conoscenze a priori pure e conoscenze a priori empiriche, certamente problematica in Kant, va però riguardata in relazione al fatto che la proposizione "ogni mutamento ha una causa" può assumere due diversi significati in relazione ad un uso diverso della concettualità discorsiva e così del concetto stesso di causalità. Proprio in riferimento a questo uso diverso, il fatto che tale principio venga utilizzato sia come esempio di proposizioni a priori pure, sia come esempio di proposizioni a priori empiriche non costituisce una contraddizione. Piuttosto, nello scandire il passaggio tra il modo diverso in cui Kant fa ricorso alla proposizione che riferisce ogni mutamento ad una causa è possibile trovare una chiave per meglio delineare il senso della rilettura kantiana del principio di causalità.

3. I. KANT, Über den Gebrauch teleologischer Prinzipien (=ÜGTP), AA 08:184.

4. KrV, A 41 B 58; cfr. anche $\mathrm{KrV}$ A 183 B 226, dove però il termine per mutamento non è "Veränderung" ma "Wechsel".

5. Cfr. KrV, A 171 B 213. Nei Primi principi metafisici della scienza della natura Kant 
Il fatto è però che la proposizione "ogni mutamento ha una causa" non rimane relegata, nel contesto della Critica della ragion pura, nello spazio minimo di un esempio citato in modo poco avveduto. Essa costituirà, anzi, nientemeno che il testo della seconda analogia dell'esperienza ${ }^{6}$ e, a dirla tutta, uno dei principi trascendentali a cui Kant fa appello come a una condizione a priori della possibilità dell'esperienza in generale ${ }^{7}$. Non si può certo dire che

torna sull'argomento in riferimento al movimento spaziale: "Per finire, faccio ancora notare che, non potendo la mobilità (Beweglichkeit) di un oggetto nello spazio essere conosciuta a priori e senza l'insegnamento dell'esperienza, essa non poté, appunto per questo, venire da me annoverata, nella Critica della ragion pura, tra i concetti puri dell'intelletto e che perciò, come concetto empirico, può trovar posto solo in una scienza della natura, quale metafisica applicata, scienza che si occupa di un concetto dato per mezzo dell'esperienza, per quanto in base a principi a priori". I. KANT, Metaphysische Anfangsgründe der Naturwissenschaft [=MAN], AA 04: 482; tr. it. Primi principi metafisici della scienza della natura, di L. GaLvaNI (Cappelli, Urbino, 1959) 27-28. Va ricordato, a questo proposito, che il movimento spaziale viene, qui, ricondotto ad una forma specifica di mutamento. Nella definizione II dei Primi principi metafisica della foronomia si legge infatti: "il movimento (Bewegung) di una cosa è il cambiamento (Veränderung) dei rapporti esterni di questa cosa rispetto a uno spazio dato" (MAN, AA 04: 482; tr. it. 28). Anche nei Prolegomeni il movimento viene annoverato tra i concetti della fisica generale "che non sono assolutamente puri ed indipendenti dalle sorgenti empiriche". Tali concetti "impediscono che essa possa dirsi una fisica assolutamente pura". I. KANT, Prolegomena zu einer jeden künftigen Metaphysik, die als Wissenschaft wird auftreten können (=Prol), AA 04: 295; tr. it. Prolegomeni ad ogni metafisica futura che vorrà presentarsi come scienza, di P. MARTINETTI (Rusconi, Milano, 1995) 109.

6. "Tutti i mutamenti (Veränderungen) accadono (geschehen) secondo la legge della connessione di causa ed effetto" (KrV, B 232). Nella prima edizione si legge: "Tutto ciò che accade (comincia ad essere), presuppone qualcosa, a cui fa seguito secondo una regola" (KrV, A 189). Si ritornerà in seguito su questa differenza di formulazione, che non è a mio avviso riducibile, come invece sostiene C. D. Broad, ad una semplice differenza verbale. Cfr. C. D. BROAD, Kants First and Second Analogies of Experience, "Proceedings of the Aristotelian Society" 26 (1925-1926) 189-210, 196.

7. "Dunque, è solo per il fatto che noi sottomettiamo le conseguenze dei fenomeni, e quindi tutti i mutamenti, alla legge della causalità, che l'esperienza stessa, e cioè la conoscenza empirica dei fenomeni, diviene possibile; ed è solo per la medesima legge, quindi, che diventano possibili gli stessi fenomeni quali oggetti dell'esperienza" (KrV, B 234). Ancora, nella Dialettica trascendentale si legge: "è una legge universale della stessa possibilità di ogni esperienza quella secondo cui tutto ciò che accade deve avere una causa" (KrV, A 533 B 561). Cfr. anche Prol, AA 04: 295; tr. it. 109. J. Kopper si riferisce alla seconda analogia dell'esperienza come al più importante tra i principi trascendentali. Cfr. J. KOPPER, Kants Zweite Analogie der Erfahrung, "Kant-Studien" 61 (1970) 1-4, 289-306, 289. Su questo argomento 
una proposizione di tale natura "presupponga principi empirici", men che meno che debba rimanere fuori dai confini di una filosofia trascendentale in ragione del fatto che conterrebbe il riferimento 'impuro' al fenomeno del mutamento.

Non è, insomma, così ovvio che la proposizione in questione rappresenti un esempio inappropriato di conoscenze pure a priori, a meno che non si voglia escludere dal novero di queste le stesse proposizioni trascendentali sulle quali si fonda ogni esperienza possibile. Del resto, il fatto che Kant, nel rispondere al rilievo sulla contraddittorietà dell'uso dell'esempio, dica che avrebbe potuto evitare l'equivoco eliminando dalla proposizione il riferimento al mutamento, non implica che il ricorso a questo esempio sia errato. Se fosse errato tutto ciò che è foriero di equivoci, l'unico modo per non errare rimarrebbe il non parlare affatto.

Non potrà trattarsi, allora, di escludere dal novero delle conoscenze pure a priori la proposizione fondamentale che ogni mutamento ha una causa. Piuttosto, si dovrà chiarire da dove tragga origine l'equivoco che vede l'utilizzo di tale proposizione tanto come esempio di conoscenze empiriche quanto come esempio di conoscenze pure. È lo stesso Kant a suggerire il modo di sciogliere il malinteso, quando afferma che il termine puro viene utilizzato nei due casi secondo "due accezioni diverse". La prima fa riferimento ad una conoscenza cui "non è mescolato (beigemischt) assolutamente nulla di empirico"". Sicché, in questo caso, la proposizione "ogni mutamento ha una causa" non è pura perché in essa si fa riferimento ad un contenuto che è ricavabile solo a posteriori dall'esperienza. La seconda acce-

cfr. anche K. Kramer, A Note on Transcendental Propositions in Kant's Critique of Pure Reason. Comment on Baum, in P. Bieri, R-P. Horstmann, L. KrüGER (eds.), On Transcendental Arguments and Science. Essays in Epistemology (Reidel Publishing Company, London, 1979) 37-43, in part. 40. Sull'importanza della seconda analogia nel sistema della filosofia critica cfr. anche H. J. PATON, Kant's Metaphysics of Experience (George Allen \& Unwin LTD, London, 1936) vol. II, 222.

8. ÜGTP, AA 08: 184 .

9. ÜGTP, AA 08: 184 . 
zione del termine "puro" qualifica, invece, un genere di conoscenza che "non dipende da nulla di empirico (von nichts Empirischem abbängig ist)". "Puro" qui non indica l'assenza di mescolanza con contenuti empirici, ma qualifica, piuttosto, una pretesa conoscitiva che l'esperienza, da sola, non è in grado di legittimare. Potremmo dire che "puro", in questa accezione, non ha un significato materiale, ma formale. Infatti, il "niente di empirico" non attiene al fatto che dalla proposizione debba sparire ogni commistione con contenuti informativi desunti dall'esperienza, ma chiama in causa un modo del tutto peculiare in cui l'esperienza si rende accessibile. Del resto, già le motivazioni che vengono rispettivamente avanzate nella prima Critica a supporto del carattere puro o non puro della proposizione in questione esprimono con chiarezza la differenza del punto di vista a partire dal quale essa viene presa in considerazione. Quando Kant riferisce la proposizione "ogni mutamento ha una causa" ad una conoscenza empirica, l'accento cade sul mutamento quale concetto che è ricavabile solo dall'esperienza. Invece, quando cita la stessa proposizione come esempio di conoscenza sintetica a priori, l'accento cade sull'uso di un concetto, il concetto di causa, il cui carattere di necessità e universalità non è in alcun modo desumibile dall'esperienza. Nel primo caso l'attenzione è rivolta ad un concetto, quello di mutamento, il cui contenuto è ricavabile per astrazione dalla percezione di qualcosa che muta, allo stesso modo in cui mediante astrazione da molteplici casi in cui un fenomeno segue ad un altro, si formula una regola generale concernente la loro possibile relazione causale, e da qui una regola, più estesa, concernente tutti gli accadimenti in generale. In questa prospettiva, la proposizione "ogni mutamento ha una causa" può esibire solo una universalità che, per dirla con Kant, si può definire "comparativa"10,

10. "L'esperienza non conferisce mai ai suoi giudizi una vera e rigorosa universalità, bensì solo un'universalità presunta e comparativa (mediante induzione), di modo che in senso proprio si dovrà dire: per quel che abbiamo percepito finora, non risulta alcuna eccezione a questa o a quell'altra regola. Se dunque un giudizio viene pensato secondo una rigorosa universalità, e cioè in modo da non concedere alcuna possibilità di eccezione, allora esso non sarà derivato dall'esperienza, ma sarà valido assolutamente a priori. L'universalità empirica è dunque soltanto un'arbitraria estensione della validità, da ciò che vale nella maggioranza dei casi a ciò che 
dovuta al fatto che la mente estende a tutti i casi simili la regola che si è riscontrata in un numero non trascurabile di esperienze. Il che non esclude che l'esperienza possa smentire quanto accaduto in precedenza, magari in presenza di variazioni che non erano state previste nel processo di formulazione della regola. Di più, la regola stessa, in quanto regola desunta dalla semplice esperienza, non può trovare nell'esperienza stessa una giustificazione adeguata rispetto alla pretesa, contenuta nella proposizione "ogni mutamento ha una causa", di valere in modo necessario in riferimento ai fenomeni in generale.

Il che vuol dire, in ultima analisi, che affermare che ogni mutamento ha una causa corrisponderebbe al lanciare all'esperienza una sfida che l'esperienza stessa non può raccogliere fino in fondo. Detto altrimenti, la suddetta proposizione esprime un principio il cui contenuto di necessità e universalità non è desumibile per astrazione dall'esperienza. Potremmo dire che la pretesa metafisica espressa dalla proposizione "ogni mutamento ha una causa" non è desumibile analiticamente dal concetto di ciò che muta, proprio perché non è contenuta nella mera casistica empirica del mutare delle cose, o, se si preferisce non è già contenuta nelle nostre rappresentazioni di ciò che muta considerate come cose in se stesse ${ }^{11}$. Al contrario, tale pretesa aggiunge alla mera rappresentazione di ciò che muta, e dunque al concetto empirico di mutamento, qualcosa di nuovo; sicché il principio secondo cui ogni mutamento ha una causa costituisce a

vale in tutti i casi, come avviene per esempio nella proposizione: tutti i corpi sono pesanti. Laddove invece a un giudizio appartenga essenzialmente un'universalità rigorosa, essa rivelerà una fonte particolare di conoscenza a priori" $(\mathrm{KrV}, \mathrm{B} 3-4)$.

11. "Che tutto ciò che accade abbia una causa, non può per nulla essere inferito in base al concetto di ciò che accade in generale" (KrV, A 301 B 357). L'ammonimento kantiano di non scambiare i fenomeni per le cose in se stesse non va letto in questo senso come se $\mathrm{i}$ fenomeni costituissero un'immagine semplicemente soggettiva di cose che starebbero fuori dal nostro orizzonte conoscitivo. Ben altrimenti, la critica kantiana della pretesa di accedere alle cose in se stesse fa perno sulla necessità di identificare una regola che consenta di discernere in seno alla successione delle nostre percezioni ciò che dipende da un mutamento reale. In altre parole scambiare i fenomeni per cose in sé significa per Kant pensare che le percezioni in se stesse, ovvero senza il riferimento ad una regola oggettiva della connessione dei fenomeni, possano dirci qualcosa su mutamenti ed eventi che accadono indipendentemente da noi. 
rigore una proposizione sintetica e non analitica, nel senso che la sua pretesa di necessità e universalità non è ricavabile per astrazione, dall'esperienza. Ciò significa che il principio in questione ha una natura sintetica e un'origine a priori ${ }^{12}$.

Così, quando cita la proposizione "ogni mutamento ha una causa" come esempio di conoscenze pure a priori, Kant intende evidenziare la possibilità di un uso della concettualità discorsiva che, proprio in ragione della sua pretesa di universalità e necessità, non può essere legittimato dalla semplice esperienza, in quanto anzi dell'esperienza deve costituire il piano di fondazione ${ }^{13}$.

Il testo della prima Critica è in proposito chiaro ed esplicito:

Ma, anche senza ricorrere a simili esempi per dimostrare la
realtà effettiva, nella nostra conoscenza, di puri principi fon-
damentali a priori, si potrebbe provare che essi sono indispen-
sabili per la possibilità stessa dell'esperienza, e così dimostrarli
a priori. Da dove mai, infatti, la stessa esperienza potrebbe at-

12. Ł̀ esattamente questo il senso in cui Kant nel $\$ 10$ dell'Analitica dei concetti parla di un "contenuto trascendentale" che l'intelletto porta (bringt) nelle rappresentazioni mediante l'unità sintetica del molteplice dell'intuizione in generale (Cfr. KrV, A 79 B 105). La questione della sintesi pura a priori non può, cioè, essere adeguatamente compresa, se la si legge nei termini di un incremento conoscitivo che verrebbe dall'aggiungersi del materiale empirico al concetto, inteso come mera forma vuota. Questa idea di sintesi risulta fuorviante ai fini di una corretta comprensione di ciò che Kant intende per conoscenza trascendentale, e risulta per di più inadeguata ai fini di una corretta comprensione di quel che Kant intende per concetto, e in particolare per concetto puro. L'aggiunta sintetica che qualifica il valore conoscitivo di una rappresentazione riguarda infatti, nel caso della sintesi pura a priori, il contenuto di universalità e di necessità che rende tale rappresentazione condivisibile con ogni soggetto dotato di ragione; contenuto la cui accessibilità, nel caso specifico della seconda analogia, concerne, come si vedrà, la possibilità che la successione delle nostre rappresentazioni sia comprensibile secondo una regola oggettiva della connessione dei fenomeni.

13. Si tratta esattamente di quell'uso la cui legittimità può essere accertata per Kant solo in sede di deduzione trascendentale dei concetti puri dell'intelletto: "Sennonché, tra i diversi concetti che costituiscono il tessuto così vario della conoscenza umana, se ne danno alcuni che sono determinati anche per un uso puro a priori (in modo del tutto indipendente dall'esperienza), e la legittimità di questo loro potere ha sempre bisogno di una deduzione. Per poter giustificare un tale uso, infatti, non sono sufficienti le prove attinte dall'esperienza: piuttosto, si dovrà sapere in che modo questi concetti possano riferirsi agli oggetti, pur senza ricavare questi oggetti da alcuna esperienza" (KrV, A 85 B 117). 
tingere la sua certezza, se tutte le regole in base alle quali essa procede fossero pur sempre delle regole empiriche, e perciò contingenti, di modo che difficilmente le si potrebbe far valere come dei principi primi? ${ }^{14}$

Qui, il punto di vista dell'indagine kantiana si volge ad una prospettiva più ampia di quella che concerne la conoscenza di oggetti specifici, siano essi i fenomeni empirici o anche gli oggetti matematici.

Il caso della proposizione "ogni mutamento ha una causa" assolve in questa nuova prospettiva ad una funzione emblematica. Essa può, da un lato, essere utilizzata come principio che mette in relazione eventi diversi, come quando si afferma che il vento ha fatto cadere una tegola dal tetto di una casa. Il suo significato muta, però, radicalmente quando ci si muove nell'ambito di un'indagine sulle condizioni di possibilità dell'esperienza. In quest'ambito il concetto di causa, e con esso il principio di causalità, assumono rilievo non in relazione al piano fisico della conoscenza diretta dei fenomeni empirici, ma in relazione al piano ontologico di quella conoscenza che riferisce la percezione di un accadimento a qualcosa di realmente esistente.

L'uso del concetto di causa non può esaurirsi qui, come è evidente, nel porre direttamente in relazione fenomeni diversi, come quando, ad esempio, il fisico scopre ed enuncia una legge sulla base di un'ipotesi confortata dalla osservazione empirica di diversi casi simili ${ }^{15}$. Il riferimento alla causalità svolge, piuttosto, un ruolo ad

\section{4. $\mathrm{KrV}, \mathrm{B} 5$.}

15. "la fisica può ammettere come universali alcuni principi in base alla testimonianza dell'esperienza, sebbene questi principi, se devono valere universalmente in senso stretto, dovrebbero essere derivati da fondamenti a priori”. I. KanT, Die Metaphysik der Sitten, AA 06: 215; tr. it. Metafisica dei costumi, di G. LANDOLFI Petrone (Bompiani, Milano, 2006) 29. Del rapporto tra il principio trascendentale della causalità e le leggi particolari di causalità conosciute mediante l'esperienza si è occupato in modo specifico H. E. Allison, Causality and Causal Laws in Kant: A Critique of Michael Friedman, in P. PARRINI (a cura di), Kant and Contemporary 
un livello già più profondo del nostro fare esperienza. Esso risulta, infatti, implicato quale condizione della possibilità stessa che la percezione di un accadimento si renda riconoscibile come reale e non semplicemente riconducibile ad un mutamento del nostro stato soggettivo. Come meglio si vedrà, la funzione del principio di causalità non concerne, in questo caso, una spiegazione che riconduce determinati eventi ad una causa. Piuttosto, la causalità diventa rilevante in riferimento alla possibilità stessa che si faccia esperienza di qualcosa di mutevole come di qualcosa che accade realmente nel tempo:

Mediante la semplice percezione resta indeterminata la relazione oggettiva dei fenomeni che si succedono l'uno all'altro. Ora, perché questa relazione venga conosciuta in maniera determinata, si deve pensare la relazione tra i due stati in maniera tale che, per suo tramite, venga determinato necessariamente quale dei due stati debba essere posto prima e quale dopo, senza possibilità di invertirli. Ma un concetto che comporti la necessità dell'unità sintetica può essere solo un concetto puro dell'intelletto, tale che non si trova nella percezione: in questo caso sarà il concetto della relazione di causa ed effetto, lì dove la prima determina il secondo, nel tempo, come conseguenza e non come qualcosa che, nella semplice immaginazione, potrebbe anche essere precedente ${ }^{16}$.

Dunque, è sulla base della riconducibilità della successione delle nostre percezioni ad una regola secondo la quale qualcosa segue necessariamente da ciò che precede, che si rende in generale possibile riferire l'esperienza di un accadimento a qualcosa che accade, a qualcosa che si verifica, indipendentemente dal fatto che ne facciamo esperienza. Ovvero, l'esperienza di qualcosa che muta trova la sua giustificazione oggettiva non nella semplice successione di

Epistemology (Springer, Dordrecht, 1994) 291-307. Sull'argomento cfr. anche A. BreITENBACH, Kant on Causal Knowledge. Causality, Mechanism and Reflective Fudgment, in K. Allen, T. Stoneham (eds.), Causation and Modern Philosophy (Routledge, London, 2011) 201-219.

16. KrV B 234. 
percezioni, ma nel fatto che tale successione obbedisce ad una regola che non posso variare a piacimento:

Quando, dunque, sperimentiamo (erfabren) che qualcosa accade, presupponiamo sempre che qualcosa preceda, a cui ciò che accade segua secondo una regola. Senza di ciò, infatti, io non potrei dire di un oggetto che esso segue, poiché la semplice successione nella mia apprensione, se non è determinata da una regola rispetto a qualcosa che la precede, non giustifica alcuna successione nell'oggetto. Dunque, accade sempre in riferimento a una regola - secondo la quale i fenomeni nella loro successione, ossia così come accadono, sono determinati tramite lo stato precedente- che io rendo oggettiva la mia sintesi soggettiva (dell'apprensione); e anzi, è unicamente in base a questa presupposizione che è possibile l'esperienza di qualcosa che accade ${ }^{17}$.

La proposizione "ogni mutamento ha una causa" non vale, dunque, solo come criterio di ricerca o come legge di spiegazione di una determinata relazione tra due o più eventi, ma costituisce, più radicalmente, un principio a partire dal quale la percezione di un accadimento si rende legittimamente riferibile ad un cambiamento temporale di stato che avviene nell'oggetto e non semplicemente nel soggetto percipiente. Il concetto di causalità si traduce, così, nello schema della successione temporale secondo una regola. Il che significa che esso non esprime letteralmente una relazione causale tra eventi diversi, come quando si pone in relazione il sorgere del sole con il riscaldamento di una pietra, ma concerne, più radicalmente, la modalità fondamentale del nostro poter renderci coscienti di essere al cospetto di qualcosa che muta fuori di noi in ragione del fatto che la successione delle percezioni mediante la quale facciamo esperienza di questo mutamento segue un ordine temporale irreversibile. Sicché siamo autorizzati a pensare e ad affermare che l'accadimento che percepiamo secondo momenti successivi è reale

17. KrV, A 195 B 240. 
e non dovuto alla semplice successione dei nostri stati percettivi. Detto diversamente: si dà un uso del concetto di causa come principio costitutivo, come quando poniamo in relazione eventi successivi riferendo ad uno l'esser causa e all'altro l'essere effetto che da quella causa segue. Tale uso costitutivo però, applicato al concetto generale di mutamento, segna solo un'estensione arbitraria della conoscenza da ciò che vale per la maggioranza dei casi a ciò che vale in tutti i casi. Così che la proposizione "ogni mutamento ha una causa" può vantare al più una universalità comparativa, mentre non può soddisfare un'istanza a priori di necessità e universalità rigorosa. Da questo punto di vista Kant non è incoerente quando afferma che la proposizione "ogni mutamento ha una causa" va annoverata tra le conoscenze a priori ma empiriche. A priori è la pretesa di necessità e universalità rigorosa espressa in una proposizione che attribuisce ad ogni mutamento una causa, empirica è l'universalità di una regola che viene analiticamente ricavata dalla mera comparazione di diversi casi simili.

Il che significa, in definitiva, che la proposizione "ogni mutamento ha una causa" esprime una pretesa a priori di necessità e universalità che, però, l'esperienza non può garantire in modo adeguato, almeno fino a quando si rimane fermi all'uso costitutivo della causalità come principio che applichiamo, nell'ambito della conoscenza empirica, direttamente ad una serie successiva di eventi.

Si dà però, per Kant, anche un uso regolativo del principio di causa $^{18}$, compatibile stavolta con le pretese metafisiche di una conoscenza pura a priori. La possibilità di tale uso fa sì che la stessa proposizione "ogni mutamento ha una causa" possa essere annoverata, in contraddizione solo apparente con quanto Kant affermava poco prima, tra i giudizi puri a priori.

Nel suo uso regolativo il riferimento alla causalità opera come un esemplare di relazione che qualifica in modo determinato, ovvero secondo una regola, ciò che nel tempo segue rispetto a ciò che pre-

18. Sulla distinzione tra regolativo e costitutivo in relazione alle analogie dell'esperienza cfr. S. TAKEDA, Kant und das Problem der Analogie. Eine Forschung nach dem Logos der Kantischen Philosophie (Nijhoff, Den Haag, 1969) 72. 
cede, così che ciò che segue non può essere invertito con ciò che precede senza che la relazione stessa non perda di significato. Ora, proprio la regola che impedisce di invertire ciò che segue con ciò che precede consente di discernere in seno alle nostre percezioni il riferimento a qualcosa che muta realmente. In altri termini, noi siamo autorizzati a riferire percezioni che si succedono nel tempo al mutare reale delle cose solo nella misura in cui le diverse percezioni che compongono l'esperienza di un accadimento sono riferibili ad una regola secondo cui ciò che segue nel tempo risulta necessariamente determinato da ciò che precede. Il che, si badi, non significa affermare che l'uno sia causa dell'altro; piuttosto significa sostenere che l'irreversibilità di una successione temporale tra fenomeni può essere pensata in analogia con la regola che sta alla base di una relazione causale, dal momento che, come nella relazione causale, non è possibile invertire nel tempo ciò che segue con ciò che precede. E difatti, che la proposizione "ogni mutamento ha una causa" figuri tra le analogie dell'esperienza significa che la sua applicazione come principio puro a priori non concerne la conoscenza diretta dei fenomeni empirici: "i fenomeni, afferma Kant, non devono essere sussunti immediatamente sotto le categorie, ma sotto i loro schemi soltanto" 19 . E lo schema della causalità "consiste [...] nella successione del molteplice, in quanto essa è soggetta ad una regola"20.

La legge della causalità può, infatti, valere come principio puro della determinazione dei fenomeni, non in quanto regola ricavata dall'osservazione empirica di diversi casi simili, ma solo in quanto proposizione contenente la modalità in cui si rende traducibile in un linguaggio logico, e dunque in linea di principio condivisibile con ogni soggetto dotato di ragione, quel che sul piano sensibile si mostra come una successione non arbitraria di percezioni, ovvero

19. KrV, A 181 B 223. Alla determinazione della causalità come "principio" corrisponde il fatto che l'intelletto, come dice Kant, "[...] non è soltanto la facoltà delle regole riguardo a ciò che accade, ma è anche la fonte dei principi, quella fonte secondo la quale tutto (nel senso di tutto quello che ci si può presentare come oggetto) sottostà necessariamente a delle regole, poiché senza queste regole ai fenomeni non potrebbe mai spettare la conoscenza di un oggetto ad essi corrispondente" (KrV, A 158-159 B 197-198).

20. KrV, A 144 B 183. 
il fenomeno del mutamento ${ }^{21}$, "il che significa — come dice Kantche non posso disporre l'apprensione se non precisamente in questa successione"22, così che la stessa possibilità della percezione di un mutamento si lega necessariamente ad un determinato ordine nell'apprensione ${ }^{23}$.

Analogia dell'esperienza significa, allora, che il principio di causalità costituisce nel pensiero l'analogo di quel che nel sensibile viene rappresentato come una successione temporale irreversibile, e per questo non arbitraria ${ }^{24}$. E pensare l'irreversibilità di una succes-

21. Così Kant a proposito delle analogie dell'esperienza in generale: "Grazie a questi principi, dunque, saremo autorizzati a congiungere i fenomeni con l'unità logica e universale dei concetti, ma solo secondo un'analogia; e perciò, se è vero che nello stesso principio ci serviremo della categoria, tuttavia nella realizzazione di quest'ultima (e cioè nella sua applicazione ai fenomeni), al posto del principio metteremo lo schema della categoria, quello che costituisce la chiave del suo uso, o meglio: metteremo questo schema a lato della categoria, come sua condizione restrittiva, e lo chiameremo formula del principio" (KrV, A 181 B 224).

22. KrV, A 194 B 238.

23. A partire da questa prospettiva mi pare perda di forza la nota obiezione di Stawson secondo cui Kant, nella seconda analogia, commetterebbe addirittura un "non sequitur di grossolanità paralizzanti”, passando dalla necessità che caratterizza il senso di una successione non arbitraria della nostra apprensione nel caso della percezione di un mutamento, alla proposizione secondo cui questo stesso mutamento deve essere concepito come necessario; ovvero a quella necessità che implica il riferimento ad una legge della determinazione causale. P. F. STRAWSON, Saggio sulla "Critica della ragion pura", tr. it. di M. PALUMBO (Laterza, Roma-Bari, 1985) 124-125. Questa obiezione descrive un iter argomentativo che, se per certi versi può essere suggerito da qualche passo della seconda analogia, di questa non identifica affatto la ratio probandi. L'argomento di Kant non segna, infatti, il passaggio dalla necessità implicata nel carattere non arbitrario della successione apprensiva che forma la percezione di un mutamento alla necessità espressa dalla legge fisica sul carattere causale delle relazioni tra i fenomeni. Piuttosto, la causalità assume, come si è visto, un ruolo all'interno dell'Analitica dei principi, e dunque una validità in quanto principio trascendentale, in relazione al fatto che essa fornisce lo strumento concettuale per codificare in un linguaggio logico-discorsivo il dato fenomenico di una successione temporale non reversibile, così che questo carattere non reversibile possa essere tradotto in termini di necessità logica. Il principio di causalità, e la sua validità dal punto di vista trascendentale, non indicano altro che la modalità sintetico-discorsiva in cui si articola la possibilità di divenire coscienti di un mutamento esterno.

24. Da questo punto vista sembra ancora alquanto vaga la spiegazione fornita da Pederson, secondo cui le analogie dell'esperienza "sono analogie [...] nel senso che si applicano analogamente a varie situazioni concrete". D. PEDERSON, Causality and Objectivity: The Arguments in Kant's Second Analogy, "Aporia” 21/1 (2011) 14. 
sione temporale secondo la regola contenuta nel concetto di causa è il modo in cui ci rendiamo comprensibile il succedersi dei fenomeni in quanto riferibile ad uno stato oggettivo di cose e non dipendente dal fatto che ne facciamo esperienza. Il pensiero contenuto nel concetto di causalità deve poter accompagnare la rappresentazione di qualcosa che muta quando la rappresentazione denota effettivamente qualcosa che muta realmente fuori di noi. In riferimento alla possibilità di un uso puro del concetto di causa la questione decisiva non è allora, per Kant, se ad una determinata successione di eventi sia o no applicabile il concetto di causa e neppure se a tutti i fenomeni in generale vada attribuita una causa. Piuttosto, si tratta di comprendere che ogni volta che ci troviamo in presenza di un mutamento di stato la cui realtà non mettiamo in dubbio, possiamo far ciò in ragione del fatto che la successione temporale delle nostre percezioni risulta comprensibile secondo una regola che alle nostre percezioni assegna una precisa posizione nel tempo. Questa regola è appunto quella espressa dalla causalità, o meglio, non direttamente dalla causalità, ma da ciò che della causalità rappresenta la nuda ratio schematica, ovvero, l'ordine determinato di una successione in cui ciò che segue nel tempo non può essere invertito con ciò che precede ${ }^{25}$.

25. Limitatamente a questo aspetto mi pare che la ratio probandi della seconda analogia dell'esperienza trovi espressione più adeguata nella formulazione della prima edizione: "Tutto ciò che accade (comincia ad essere), presuppone qualcosa, a cui fa seguito secondo una regola" (KrV, A 189). Qui Kant non fa, infatti, riferimento diretto alla causalità, come invece farà nella seconda edizione, mentre richiama in modo esplicito la regola, contenuta nel concetto di causalità, in base alla quale è possibile determinare un ordine nella successione dei fenomeni. Ed è esattamente in quanto fornisce la regola per la determinazione dei fenomeni nel tempo che la causalità svolge un ruolo decisivo nell'ambito dell'Analitica dei principi. La formulazione della seconda edizione risulta, invece, preferibile a quella della prima in relazione al fatto che in essa si parla in modo esplicito di mutamenti ("Tutti i mutamenti accadono secondo la legge della connessione di causa ed effetto" $[\mathrm{KrV}$, B 232]). Ciò richiama, infatti, la tesi della prima analogia dell'esperienza secondo cui ogni accadimento è riconducibile ad un mutamento sostanziale, dunque ad una alterazione. Su questo aspetto concordo con le osservazioni di P. GUYER, Kant and the Claims of Knowledge (Cambridge University Press, New York-New Rochelle-Melbourne-Sidney, 1987) 239. Si avrà modo di tornare sulla centralità del concetto di mutamento nell'ambito della seconda analogia dell'esperienza (cfr. infra, 502-504). 
Il principio di causalità esprime in questo senso, nei termini della cogenza logica del pensiero, l'accadere di qualcosa fuori di noi su cui il nostro arbitrio non ha alcun potere.

L'interesse di Kant è, in tal modo, quello di cogliere nel concetto di causa, e così nel principio di causalità, il modello, meglio lo schema di lettura e di codifica di una successione di eventi che giudichiamo come oggettiva e dunque distinguiamo dal semplice succedersi delle nostre percezioni, per il fatto che tale successione segue una regola della connessione dei fenomeni.

Come nel celebre esempio che occorre nella seconda analogia, quando vedo una nave che scende lungo la corrente, non posso invertire a piacimento l'ordine di successione delle mie rappresentazioni, l'apprensione di ciò che precede con l'apprensione di ciò che segue, non posso cioè decidere che la percezione della nave nella parte più bassa del fiume preceda la percezione della stessa nave nella parte più alta. In questo caso l'apprensione successiva dell'oggetto rimanda ad una regola oggettiva della connessione dei fenomeni, tale che la percezione del mutamento non risulta dal semplice fatto che i fenomeni vengono appresi l'uno dopo l'altro (fatto comune anche alla percezione di una casa ben piantata sul terreno $)^{26}$, ma acquista il significato di qualcosa che reca realmente in sé il susseguirsi di stati differenti nell'oggetto percepito. In questo caso, cioè, la successione soggettiva dell'apprensione, ovvero, la regola secondo la quale si susseguono le nostre percezioni, non potrà che essere dedotta dalla successione oggettiva dei fenomeni ${ }^{27}$.

26. "L'apprensione del molteplice del fenomeno è sempre successiva" (KrV, B 234). "Nella sintesi dei fenomeni il molteplice delle rappresentazioni procede sempre in successione. Con ciò non viene rappresentato alcun oggetto, poiché attraverso questa successione, che è comune a tutte le apprensioni, nessuna cosa si distingue da un'altra. Ma non appena percepisco, o presumo, che in questa successione vi sia una relazione con lo stato precedente, dal quale la rappresentazione segue secondo una regola, qualcosa verrà rappresentato come un accadimento, ossia come qualcosa che sta accadendo, e cioè consocerò un oggetto, che devo porre nel tempo in un qualche punto determinato, il quale non può essergli assegnato altrimenti, tenendo conto dello stato precedente" (KrV, A 198 B 243).

27. Cfr. KrV, A 193 B 238. La distinzione tra tempo soggettivo e tempo oggettivo, certamente funzionale agli argomenti addotti nella seconda analogia, non toglie però il fatto che anche la mera successione soggettiva delle nostre rappresenta- 
L'uso schematico del concetto di causa, inteso come rappresentazione di una regola della successione non arbitraria delle nostre percezioni, consente, così, di distinguere nella percezione il tempo oggettivo dal semplice succedersi di diverse impressioni sensibili nel nostro animo, e di riferire la percezione di un mutamento al mutare effettivo, all'accadere reale, di qualcosa nel tempo:

Si tratta quindi di mostrare, mediante degli esempi, che noi non attribuiamo mai, neppure nell'esperienza, la successione [...] all'oggetto, distinguendo tale successione da quella soggettiva della nostra apprensione, se non quando vi sia come fondamento una regola, che ci costringa a osservare quest'ordine delle percezioni piuttosto che un altro, e che anzi è proprio questa costrizione ciò che rende anzitutto possibile la rappresentazione di una successione nell'oggetto ${ }^{28}$.

Il riferimento al tempo e alla sua determinabilità secondo il principio della causalità è decisivo per una considerazione che conferisce al fenomeno empirico del mutamento un posto di rilievo nell'ambito di indagine proprio della Critica della ragion pura. La proposizione "ogni mutamento ha una causa" rientra, cioè, a pieno titolo tra le conoscenze pure a priori nella misura in cui ha come oggetto il tempo in quanto forma pura del mutamento e insieme il concetto di causa in quanto principio di determinazione del tempo:

zioni conserva sempre come sfondo la successione del tempo oggettivo. Ovvero, anche quella che Kant indica come semplice successione arbitraria delle nostre rappresentazioni, quale si darebbe nel caso in cui posso indifferentemente partire da destra o da sinistra nell'apprensione di una casa, richiede pur sempre, come condizione della sua stessa conoscibilità in quanto serie temporale, il riferimento ad un contesto di relazioni determinate e dunque, in ultima analisi, a quell'ordine regolato di connessioni tra i fenomeni che segna l'accesso conoscitivo a mutamenti esterni. La direzione argomentativa che affiora dalla seconda analogia, per non dire dall'intero impianto della fondazione critico-trascendentale dell'esperienza, sembra proprio voler confutare ogni punto di vista psicologico che muova da un presunto ordine temporale primitivo interno per poi guadagnare l'ordine successivo dei mutamenti esterni, e ciò perché l' "interno" è, nella prospettiva kantiana, già sempre parte di un contesto mondano di relazioni fenomeniche regolate secondo leggi.

28. KrV, A 196-197 B 241-242. 
Ma la forma di ogni mutamento, la sola condizione alla quale esso può procedere come il sorgere di un altro stato (quale che sia il suo contenuto, cioè lo stato che viene modificato), e quindi la successione degli stati stessi (l'accaduto), è qualcosa che può $[. .$.$] essere considerato a priori secondo la legge della$ causalità e le condizioni del tempo ${ }^{29}$.

\section{4}

Il tema della determinabilità del tempo in quanto forma pura dell'intuizione mediante la regola contenuta nel concetto di causalità è ovviamente centrale nel contesto di un argomento che mira a stabilire i principi a priori della possibilità dell'esperienza, e che identifica nella legge di causa ed effetto non semplicemente una regola da applicare direttamente a determinate successioni di eventi, $\mathrm{ma}$, in radice, il principio che consente di discernere in seno alla successione delle nostre percezioni il riferimento oggettivo ad un mondo che muta fuori di noi. Il che significa che quando Kant parla di causalità, egli non è interessato unicamente alla successione temporale di eventi differenti, quale può essere ad esempio la relazione causale che posso individuare tra il vento che soffia e una tegola che mi cade sulla testa. Anche l'immagine della nave che scende lungo la corrente, che nella seconda analogia Kant portava ad esempio di una successione non arbitraria, non riguarda la connessione causale-temporale di eventi differenti, ma insiste su un cambiamento da riferire in generale ad uno stato di cose (la nave, il fiume, la corrente etc.). Lo stesso concetto di evento, inteso come qualcosa che accade, non può che essere percepito, secondo Kant, nei termini del mutare di qualcosa, o meglio, del mutare di uno stato di cose:

Che qualcosa accada, cioè divenga qualcosa oppure uno stato che precedentemente non era, non può essere percepito

29. KrV, A 207 B 252. 
empiricamente, lì dove non preceda un fenomeno che non contenga in sé questo stato ${ }^{30}$.

La tesi kantiana che riconduce ogni forma dell'accadere ad un mutamento di stato richiederebbe un discorso ben più articolato di quello che può essere svolto nello spazio della presente trattazione. Se non altro perché chiama in causa lo stretto legame che sussiste tra la prima e la seconda analogia dell'esperienza. Se, infatti, la seconda analogia afferma che "tutti i mutamenti accadono secondo la legge della connessione di causa ed effetto", nella prima si stabilisce che "tutti quanti i fenomeni della successione temporale sono soltanto mutamenti" 31 , ovvero, qualsiasi successione temporale va sempre ricondotta ad un cambiamento di stato ${ }^{32}$.

Non posso soffermarmi su questo aspetto dell'Analitica dei principi. Ciò che invece merita di essere evidenziato, nel contesto di questo lavoro, è il fatto che l'argomento della seconda analogia, lo stretto legame con la prima, e la conseguente riconduzione di ogni successione temporale a quella concernente un cambiamento di stato, insiste su un uso del principio di causalità come regola che, prima di riguardare la connessione empirica di eventi diversi nel tempo, costituisce la condizione che consente di riconoscere il $m u$ tare di qualcosa in generale ${ }^{33}$. Questo aspetto colloca la causalità,

30. KrV, A 191 B 236-237.

31. KrV, B 232.

32. Sul concetto di cambiamento nel contesto della prima analogia dell'esperienza cfr. i rilievi di K. CRAMER, Kant's Definition of the Concept of Change, in P. LABERGE, F. Duchesneau, B. E. Morrisey (eds.), Actes du Congrès d'Ottawa sur Kant dans les traditions anglo-americaine et continentale tenu du 10 au 14 octobre 1974 (Editions de l'Université d'Ottawa, Ottawa, 1976) 364-381.

33. Su questo punto insiste B. RANG, Naturnotwendigkeit und Freibeit. Zu Kants Theorie der Kausalität als Antwort auf Hume, "Kant-Studien" 81/1 (1990) 24-56, in part. 34, 39. Già nella prima metà del Novecento la critica aveva posto l'accento sulla centralità del motivo del cambiamento sostanziale nel contesto della seconda analogia. Cfr. al riguardo i rilievi di SwABEY, Kant's Analogies of Experience, "The Philosophical Review" 31/1 (1922) 41-57, 52-53. Se l'esame della prima analogia risulta essenziale per chiarire il modello kantiano di causalità in quanto riconducibile alla condizione del mutamento sostanziale, altrettanto decisivo risulta però l'apporto che la terza analogia può dare ai fini di una descrizione più dettagliata di tale modello. E. Watkins ha posto l'accento su questo punto, facendone uno dei cardini di una lettura tesa a sottolineare in Kant il ricorso ad un paradigma 
intesa come principio che fornisce la regola di una successione non arbitraria delle percezioni, ad un livello più profondo della nostra esperienza; più profondo di quello empirico in cui il concetto di causalità viene applicato alla successione di eventi differenti. Ed è a questo livello più profondo che la proposizione "ogni mutamento ha una causa" rientra con pieno diritto tra le proposizioni pure a priori. Si tratta, infatti, di quel principio trascendentale in forza del quale prende forma oggettiva il tempo in quanto condizione di ogni successione possibile. Come dire, il concetto di causalità conosce un'applicazione che precede ogni applicazione determinata, empirica. Questa "prima applicazione" 34 connota l'uso puro, categoriale, del concetto di causa quale principio che rende possibile riconoscere accadimenti e mutamenti reali nel tempo, e con ciò rende possibile l'esperienza in generale.

di causalità non compatibile con quello che concerne la descrizione di una relazione di due o più eventi di cui l'uno venga identificato come causa dell'altro. Secondo Watkins il significato dell'attività causale non è riconducibile, infatti, ad un evento temporalmente determinato che darebbe origine all'evento successivo, ma alla capacità fondamentale che una sostanza ha di agire sull'altra in base alla propria natura, producendo in essa un mutamento e dunque una successione di stati. Sicché, l'accento non cade sulla successione tra causa ed effetto, ma sulla successione che si produce nell'effetto, negli stati di una determinata sostanza, a partire dalla attività di causazione esercitata da un'altra sostanza. In tal modo il significato ultimo dell'attività causale, o di quella che Kant chiama "causalità della causa", andrebbe cercata a un livello di realtà più profondo di quello descritto dagli eventi spazio-temporalmente determinati, i quali semmai dell'azione causale sono il risultato. Cfr. E. WATKINS, Kant and the Metaphysics of Causality (Cambridge University Press, Cambridge, 2005) in part. 185-297. La lettura di Watkins ha senza dubbio il merito di porre in rilievo, seguendo un percorso interpretativo originale, il significato metafisico-ontologico e non semplicemente epistemologico delle Analogie dell'esperienza. Tuttavia mi pare che essa rischia di non dare il giusto rilievo al significato che la causalità assume in Kant in relazione a quell'uso schematico che, come abbiamo visto, rappresenta il vero elemento di novità rispetto alla tradizione metafisica.

34. Kant parla in particolare di "prima applicazione dell'intelletto agli oggetti dell'intuizione possibile per noi" identificandola come "il fondamento di tutte le altre applicazioni”, e collegandola, com'è noto, alla funzione svolta dalla sintesi trascendentale dell'immaginazione, che è la sintesi mediante la quale il tempo, in quanto forma pura dell'intuizione sensibile, viene determinato in conformità alle categorie (Cfr. KrV, B 152). 
Allora, se da un lato la proposizione "ogni mutamento ha una causa" può valere come massima che orienta la conoscenza empirica di questa o quella determinata successione temporale tra fenomeni; dall'altro, però, la stessa proposizione costituisce il principio di intelligibilità della successione temporale come relazione di percezioni il cui succedersi obbedisce ad una regola che non dipende dall'arbitrio del soggetto percipiente, così che tutti i fenomeni che si verificano in conformità con questa regola possono legittimamente essere riferiti a mutamenti reali.

Da questo punto di vista, la proposizione "ogni mutamento ha una causa" va intesa come un principio di leggibilità dell'esperienza, a partire dal quale, soltanto, l'esperienza è resa capace di testimoniare il verificarsi di qualcosa la cui esistenza non dipende da noi, come non dipende dalla nostra percezione che una nave risalga lungo il fiume anziché andare nella direzione opposta. In ragione di ciò la proposizione "ogni mutamento ha una causa" assume un significato puro a priori. Il che non vuol dire in alcun modo, giova ripeterlo, che la pretesa a priori della causalità faccia riferimento al contenuto "dogmatico" di una proposizione che, muovendo dal semplice concetto di mutamento, deduce che ogni mutamento ha una causa ${ }^{35}$. Che ogni mutamento ha una causa non è per Kant ricavabile in modo analitico dalla nozione di ciò che muta. Se così fosse, tornerebbe l'obiezione kantiana secondo cui quello di mutamento è un concetto che implica sempre il riferimento ad un contenuto empirico, e sotto questo rispetto non è possibile che la proposizione "ogni mutamento ha una causa" rientri tra le conoscenze pure a priori.

35. "Se avessimo voluto dimostrare queste analogie in modo dogmatico, ossia in base a concetti —affermando cioè che tutto quel che esiste lo si trova solo in ciò che è permanente; che ogni accadimento presuppone qualcosa nello stato precedente a cui esso segue secondo una regola [...] - , tutta la fatica sarebbe stata completamente inutile. Infatti, mediante meri concetti di queste cose non si può pervenire da un oggetto e dalla sua esistenza all'esistenza o al modo di esistere di un altro oggetto, in qualsiasi modo li si voglia analizzare" (KrV, A 216-217 B 263-264). 
Tuttavia, la stessa proposizione, quando venga riguardata come principio a partire dal quale è possibile riconoscere in un mutamento l'accadere di qualcosa che non dipende unicamente dal modo in cui ne facciamo esperienza, dunque l'accadere di qualcosa di reale, quando cioè la proposizione "ogni mutamento ha una causa" venga riconosciuta non come semplice replica scolastica del principio nibil est sine ratione ${ }^{36}$, ma come principio trascendentale, allora essa può vantare validità pura a priori. Ciò nella misura in cui senza il riferimento alla regola della causalità sarebbe impossibile distinguere l'esperienza del mutare reale delle cose dal succedersi dei nostri stanti interni ${ }^{37}$. In questa cornice, anzi, il concetto di "mutamento" può rivelare una natura diversa da quella semplicemente riferibile al mero risultato di un'astrazione dall'empirico. E cioè, la sua riconoscibilità in quanto fenomeno oggettivo riconduce alla determinazione delle forme pure della sensibilità secondo le regole della concettualità discorsiva. Così ricompreso, ossia considerato a quel livello profondo della nostra esperienza che concerne le condizioni trascendentali della determinazione di una successione temporale oggettiva, il concetto di mutamento finisce con l'essere annoverato tra i cosiddetti "predicabili dell'intelletto", ossia tra quei concetti che, come quello di "forza", "azione" "passione" sono derivati dalla congiunzione tra le categorie e i modi della sensibilità pura, e che per questo sono, come dice Kant, "altrettanto puri" sebbene non primitivi, non originari come le categorie ${ }^{38}$.

36. Cfr. KrV, A 217 B 218, dove Kant denuncia chiaramente il carattere illusorio della pretesa di dimostrare il principio di ragion sufficiente dogmaticamente, ossia in base a semplici concetti.

37. "La dimostrazione, infatti, non mostra che il concetto dato (per esempio il concetto di ciò che accade) conduca direttamente a un altro concetto (quello di una causa), poiché un tale passaggio sarebbe un salto che non può essere affatto legittimato; essa mostra piuttosto che senza una tale connessione non sarebbe possibile la stessa esperienza, e quindi l'oggetto dell'esperienza" (KrV, A 783 B 811). Su questo punto della Disciplina della ragion pura è tornato di recente G. GORIA, Kant e la disciplina della ragion pura. Le proposizioni trascendentali sintetiche e la loro dimostrazione, "Estudos Kantianos" 3/2 (2015) 119-130, 126.

38. Cfr. KrV, A 81-82 B 107-108. Sul ruolo che la dottrina kantiana dei predicabili gioca nel rapporto tra conoscenze sintetiche a priori pure e conoscenze sintetiche a priori non pure, cfr. K. Cramer, Non-Pure Synthetic A Priori Fudgments in the 'Critique of Pure Reason', in L. WHITE BECK (ed.), Proceedings of Third International 
In tal modo, che Kant usi la stessa proposizione "ogni mutamento ha una causa" ad un tempo come esempio di conoscenze il cui contenuto empirico non è di interesse per la ricerca trascendentale, e come esempio di principi puri a priori che stanno a fondamento della stessa possibilità dell'esperienza, non può semplicisticamente venir ricondotto ad una contraddizione, come sosteneva il recensore anonimo della Critica della ragion pura, né liquidato quale mero artificio, come oggi sostiene A. Ferrarin ${ }^{39}$.

Quel che avviene è, piuttosto, un cambimento di piano che introduce ad una modalità radicalmente nuova di considerare il principio di causalità e con esso il concetto di mutamento. All'interno di questa prospettiva, come si è visto, la validità a priori della proposizione "ogni mutamento ha una causa" non si lega alla pretesa dogmatica di affermare che tutto ciò che accade ha una causa, ma dà voce all'istanza critica che nel ricorso alla regola di una successione temporale necessaria, quella stessa che è contenuta nel concetto di causa, individua la possibilità di discernere in seno alle nostre rappresentazioni il riferimento ad un mondo di mutamenti che si dà indipendentemente da noi. È in questa misura che la proposizione "ogni mutamento ha una causa" guadagna lo statuto di un "principio trascendentale", ovvero, in termini kantiani, di un principio "col quale è rappresentata la condizione universale a priori, sotto la quale soltanto le cose possono diventare oggetti della nostra conoscenza in generale" ${ }^{40}$. Un "principio trascendentale" riguarda l'esperienza dal punto di vista della sua possibilità in generale, il che significa che la causalità vale come principio puro a priori in quanto condizione fondamentale del nostro fare esperienza di successioni temporali oggettive in base alle quali si rende comprensibile il mutamento:

Kant Congress. Held at the University of Rochester, March 30-April 4, 1970 (D. Reidel, Dordrecht, 1972) 246-254, in part. 250-251.

39. Cfr. supra, nota 2.

40. I. KanT, Kritik der Urteilskraft (=KU), AA 05:181; tr. it. Critica del Giudizio, di A. GARGIUlo (Laterza, Roma-Bari, 2005) 31. 
Così il principio della conoscenza dei corpi, come sostanze e come sostanze mutevoli, è trascendentale, quando s'intenda che il loro mutare debba avere una causa ${ }^{41}$.

Un principio trascendentale, scrive dunque Kant, "[...] non contiene nulla di empirico"42, nella misura in cui "[...] il concetto degli oggetti, in quanto sono pensati come sottoposti ad esso, non è che il concetto puro di oggetti della possibile conoscenza d'esperienza in genere" ${ }^{\text {"3 }}$. Ora, è esattamente questo il senso in cui il principio di causalità può vantare lo statuto di una proposizione sintetica a priori pura. Ossia, non nel senso ingenuamente dogmatico che venga meno qualunque riferimento a contenuti che derivano dall'esperienza, ma nel senso eminentemente critico di un principio che non si rivolge al piano epistemologico della conoscenza diretta di relazioni tra oggetti specifici, ma concerne il piano ontologico delle condizioni a priori che rendono in generale possibile l'esperienza in quanto esperienza di oggetti. In questo caso, come dice Kant, è sufficiente "che il corpo sia pensato solo mediante predicati ontologici (concetti puri dell'intelletto [...] per conoscere a priori la proposizione" ${ }^{44}$. Ed è, in definitiva, in ragione di questa investitura trascendentale, che la proposizione "ogni mutamento ha una causa", rientra nello spazio di interesse della Critica della ragion pura. Laddove l'uso della stessa proposizione per la determinazione della relazione tra i fenomeni che si danno in concreto nell'esperienza implica il riferimento all'elemento contingente, empirico-fattuale, e pertanto non può essere considerato puro $^{45}$.

41. KU, AA 05:181; tr. it. 31-33.

42. Cfr. KU, AA 05: 181; tr. it. 33.

43. KU, AA 05: 181-182; tr. it. 33.

44. KU, AA 05: 181; tr. it. 33.

45. In quanto riguardano l'esperienza dal punto di vista della sua possibilità in generale i principi trascendentali vanno distinti, secondo Kant dai principi metafisici: "un principio si chiama metafisico quando esso rappresenta la condizione a priori sotto la quale soltanto oggetti, il cui concetto deve essere dato empiricamente, possono essere ulteriormente determinati a priori” (KU, AA 05: 181). Rispetto al piano della conoscenza trascendentale, i principi metafisici richiedono, cioè, una determinazione ulteriore del dominio della legislazione naturale (e così dell'uso dei concetti a priori che di tale legislazione costituiscono gli elementi fondamen- 
Rimane, ovviamente, in piedi la questione se ed entro quali limiti questo radicale cambiamento di prospettiva impresso alla nozione di causalità sia in grado di fornire, come è nelle intenzioni dell'autore della Critica della ragion pura, una fondazione dell'esperienza in generale. Il che significa, in ultima analisi, chiedersi fino a che punto Kant offra una risposta che metta davvero fuori gioco lo scetticismo di Hume in merito alla possibilità di una fondazione filosofica rigorosa della validità conoscitiva del concetto di causalità.

A rendere ancora meno agevole questa risposta è, peraltro, il fatto che Hume presenta un'impostazione del rapporto tra successione temporale e causalità ben più complessa di quella che sembra volergli concedere Kant ${ }^{46}$.

Oltretutto, un esame ravvicinato della dottrina kantiana dell'idealismo trascendentale, e dunque delle tesi dell'Estetica, rivela una concezione del tempo che entra per certi versi in frizione con quella che emerge dall'esame della seconda analogia dell'esperienza ${ }^{47}$. Basti

tali) in vista della conoscenza degli oggetti fisici. Senza entrare nel dettaglio della trama articolata che innerva questa distinzione kantiana, si può dire che la proposizione "ogni mutamento ha una causa", in quanto principio trascendentale, rientra tra le conoscenze pure a priori, mentre, in quanto principio metafisico, richiede il riferimento al piano contingente della conoscenza empirica, e per questo rientra tra le proposizioni sintetiche a priori ma empiriche. Sull'argomento cfr. L. Amoroso, Senso e consenso. Uno studio kantiano (Guida, Napoli, 1984) 62-63.

46. A. T. NUYEN — Sense, Reason and Causality in Hume and Kant, "Kant-Studien" 81/1 (1990) 57-68, 58-59—, evidenzia, ad es., come anche in Hume la percezione del cambiamento sia una impressione complessa, non immediatamente derivata da semplici impressioni sensoriali ma mediata da altre impressioni e idee, inclusa l'idea stessa di causalità. Da questo punto di vista, anzi, la posizione di Hume non sarebbe per l'Autore troppo distante da quella di Kant. Su tali basi Nuyen contesta la posizione interpretativa di G. NAGEL — Substance and Causality, in WILLIAM A. Harper, R. Meerbote (eds.), Kant on Causality, Freedom, and Objectivity (University of Minnesota Press, Minneapols, 1984 97-107, -101)—, il quale riferisce invece a Hume la concezione secondo cui la nostra coscienza muove da elementi sensoriali immediati che si danno ad essa in modo aproblematico.

47. F. Venturi, nell'intento di valorizzare il ruolo del tempo in quanto intuizione, insiste sull'idea di una "[...] separazione dei ruoli tra tempo e causa che conduce ad una ripartizione tra le successioni: quelle temporali, che possiamo definire 'successioni in generale', e quelle causali, che possiamo indicare come 'successioni necessarie"'. F. VENTURI, Gli odierni commentatori di Kant alla prova di Leibniz, “Annali del Dipartimento di filosofia" XVI (Firenze, 2010) 39-54, 46. M. Valaris evidenzia, dal canto suo, la difficoltà di far coesistere l'ipotesi di una temporalità immediata con l'idea che spazio e tempo siano prodotti della sintesi figurata 
pensare, solo per far menzione di uno dei luoghi classici della Kantforschung, ai cortocircuiti che la prima Critica sembra generare tra una teoria causale-relazionale del tempo che ne riferisce la determinabilità oggettiva alla possibilità di esibire una regola a priori delle relazioni tra i fenomeni e la tesi secondo cui le successioni temporali, in quanto intuizioni pure, non derivano dalle relazioni tra gli oggetti ${ }^{48}$.

Non è qui la sede per discutere queste questioni. Ciò che, invece, preme evidenziare è il fatto che la torsione in senso "trascendentale" che Kant imprime al concetto di causalità, valga o no come ipotesi fondativa dell'esperienza in generale, fornisce la chiave per ricondurre ad una formulazione coerente quel che ad un lettore che si fermi alla lettera delle affermazioni kantiane prese isolatamente può apparire contraddittorio.

Tra l'affermazione che la proposizione "ogni mutamento ha una causa" non è di interesse della Critica della ragion pura per via delle sue compromissioni con contenuti empirici, e il fatto che la stessa proposizione venga esplicitamente citata come esempio di conoscenze pure a priori, non vi è contraddizione. C'è solo la fatica

dell'immaginazione. M. VALARIS, Inner Sense, Self-Affection \& Temporal Consciousness in Kant's Critique of Pure Reason, "Philosophers' Imprint" 8/4 (2008) 16. In ogni caso, il fatto che il tempo, in quanto intuizione pura, si distingua costitutivamente dalla funzione intellettuale che del tempo fornisce la determinazione, non può tradursi, in Kant, nell'idea di un tempo inteso come fenomeno originario della soggettività sul quale poi si applichino estrinsecamente le operazioni dell'intelletto. Questa tesi viene, infatti, messa fuori gioco dalla peculiare impostazione della dottrina del senso interno e dell'autoaffezione. Cfr. a questo riguardo C. LA RocCA, Soggetto e mondo. Studi su Kant (Marsilio, Venezia, 2003) 53-76.

48. Sulla ipotesi di un conflitto tra due diverse concezioni del tempo che si evidenzierebbe nel passaggio dall'Estetica all'Analitica trascendentale insistono T. K. SwING, Kant's Transcendental Logic (Yale University Press, New Haven-London, 1969) 151-152; W. A. Suchting, Kant's Second Analogy of Experience, "Kant-Studien" 58/1-4 (1967) 355-369, 367-368. M. Pera, pur ammettendo la presenza di una "tensione bipolare in Kant" in riferimento alla concezione del tempo, mostra in modo circostanziato come non si dia nella prima Critica una teoria causale-relazionale del tempo in senso stretto, e soprattutto come non vi sia contraddizione "[...] tra il dire che il tempo è qualcosa e la nozione originaria di esso un'intuizione e il dire che la durata e l'ordine temporale di stati, eventi, processi, si determinano sulla base di relazioni fisiche tra di essi”. Cfr. M. PERA, Hume, Kant e l'induzione (Il Mulino, Bologna, 1982) 171-197. 
e talora anche l'ambiguità di un passaggio che Kant, tra mille difficoltà, cerca di compiere nel tentativo critico di fondare su nuove basi la validità del principio di causalità quale principio che si rende applicabile a priori ad eventi successivi nella misura in cui esprime, nella sua nuda ratio schematica, la regola in base alla quale la successione delle nostre percezioni si rende atta ad esprimere qualcosa che muta oggettivamente nel tempo.

\section{REFERENCES}

H. E. Allison, Causality and Causal Laws in Kant: A Critique of Michael Friedman, in P. PARRINI (ed.), Kant and Contemporary Epistemology (Springer, Dordrecht, 1994) 291-307.

L. Amoroso, Senso e consenso. Uno studio kantiano (Guida, Napoli, 1984).

A. Breitenbach, Kant on Causal Knowledge. Causality, Mechanism and Reflective Fudgment, in K. Allen, T. Stoneham (eds.), Causation and Modern Philosophy (Routledge, London, 2011) 201-219.

C. D. BroAD, Kants First and Second Analogies of Experience, "Proceedings of the Aristotelian Society" 26 (1925-1926) 189-210.

K. Cramer, Non-Pure Synthetic A Priori fudgments in the 'Critique of Pure Reason', in L. White BeCK (ed.), Proceedings of Third International Kant Congress. Held at the University of Rochester, March 30-April 4, 1970 (D. Reidel, Dordrecht, 1972) 246-254.

K. Cramer, Kant's Definition of the Concept of Change, in P. LABERGE, F. Duchesneau, B. E. Morrisey (eds.), Actes du Congrès d'Ottawa sur Kant dans les traditions anglo-americaine et continentale tenu du 10 au 14 octobre 1974 (Editions de l'Université d'Ottawa, Ottawa, 1976) 364-381.

A. FERRARIN, Come è possibile comprendere i giudizi sintetici a priori, in L. Amoroso, C. La RocCa. A. Ferrarin (eds.), Critica della ragione e fenomenologia dell'esperienza (ETS, Pisa, 2011) 395-414.

G. GORIA, Kant e la disciplina della ragion pura. Le proposizioni trascendentali sintetiche e la loro dimostrazione, "Estudos Kantianos" 3/2 (2015) 119-130. 
P. GuYER, Kant and the Claims of Knowledge (Cambridge University Press, New York-New Rochelle-Melbourne-Sidney, 1987).

J. Kopper, Kants Zweite Analogie der Erfabrung, "Kant-Studien", 61/1-4 (1970) 289-306.

K. Kramer, A Note on Transcendental Propositions in Kant's Critique of Pure Reason. Comment on Baum, in P. BIERI, R-P. HORSTMANN, L. KRÜGER (eds.), On Transcendental Arguments and Science. Essays in Epistemology (Reidel Publishing Company, London,1979) 37-43.

C. LA RoccA, Soggetto e mondo. Studi su Kant (Marsilio, Venezia, 2003).

G. NAgel, Substance and Causality, in W. A. Harper, R. Meerbote (eds.), Kant on Causality, Freedom, and Objectivity (University of Minnesota Press, Minneapols, 1984) 97-107.

A. T. Nuyen, Sense, Reason and Causality in Hume and Kant, "Kant-Studien" 81/1 (1990) 57-68.

H. J. Paton, Kant's Metaphysics of Experience (George Allen \& Unwin LTD, London, 1936).

D. Pederson, Causality and Objectivity: The Arguments in Kant's Second Analogy, "Aporia” 21/1 (2011) 11-24.

M. PERA, Hume, Kant e l'induzione (Il Mulino, Bologna, 1982).

B. RANG, Naturnotwendigkeit und Freibeit. Zu Kants Theorie der Kausalität als Antwort auf Hume, "Kant-Studien" 81/1 (1990) 24-56.

P. F. STRAwson, Saggio sulla "Critica della ragion pura", tr. it. di M. Palumbo (Laterza, Roma-Bari, 1985).

W. A. Suchting, Kant's Second Analogy of Experience, "Kant-Studien" 58/1-4 (1967) 355-369.

W. Curtis Swabey, Kant's Analogies of Experience, "The Philosophical Review", 31/1 (1922) 41-57.

T. K. SwING, Kant's Transcendental Logic (Yale University Press, New Haven-London, 1969).

S. Takeda, Kant und das Problem der Analogie. Eine Forschung nach dem Logos der Kantischen Philosophie (Nijhoff, Den Haag, 1969).

M. VALARIS, Inner Sense, Self-Affection, \& Temporal Consciousness in Kant's Critique of Pure Reason, "Philosophers' Imprint" 8/4 (2008) 1-18. 
PER UNA RILETTURA KANTIANA DEL PRINCIPIO DI CAUSALITÀ

F. VENTURI, Gli odierni commentatori di Kant alla prova di Leibniz, “Annali del Dipartimento di Filosofia" XVI (Firenze 2010) 3954.

H. WAininger, Commentar zur Kants Kritik der reinenn Vernunft, (Spemann, Stuttgart, 1881).

E. WATKIns, Kant and the Metaphysics of Causality (Cambridge University Press, Cambridge, 2005). 
